\title{
MODELING OF HIGH SPEED REACTING FLOWS: ESTABLISHED PRACTICES AND FUTURE CHALLENGES
}

\author{
R. A. Baurle * \\ NASA Langley Research Center, Hampton, Va 23681
}

\begin{abstract}
Computational fluid dynamics (CFD) has proven to be an invaluable tool for the design and analysis of highspeed propulsion devices. Massively parallel computing, together with the maturation of robust CFD codes, has made it possible to perform simulations of complete engine flowpaths. Steady-state Reynolds-Averaged Navier-Stokes simulations are now routinely used in the scramjet engine development cycle to determine optimal fuel injector arrangements, investigate trends noted during testing, and extract various measures of engine efficiency. Unfortunately, the turbulence and combustion models used in these codes have not changed significantly over the past decade. Hence, the CFD practitioner must often rely heavily on existing measurements (at similar flow conditions) to calibrate model coefficients on a caseby-case basis. This paper provides an overview of the modeled equations typically employed by commercialquality CFD codes for high-speed combustion applications. Careful attention is given to the approximations employed for each of the unclosed terms in the averaged equation set. The salient features (and shortcomings) of common models used to close these terms are covered in detail, and several academic efforts aimed at addressing these shortcomings are discussed.
\end{abstract}

\section{INTRODUCTION}

Computational Fluid Dynamics (CFD) models typically employed for compressible reacting internal flows have far less predictive capabilities than their counterparts used for low-speed external flow applications. CFD models and experimental techniques applied to low-speed external flows have reached a level of maturity such that commercial aviation companies are now asking for drag coefficient estimates that have an uncertainty level as low as one count ${ }^{1}$ or 0.0001 . This translates to an uncertainty level of $\pm 0.5 \%$ of the total drag for a typical aircraft at cruise conditions. While this level of accuracy

\footnotetext{
*Aerospace Engineer, Hypersonic Airbreathing Propulsion Branch, Senior Member AIAA.
}

has not yet been realized, a statistical analysis of drag predictions presented at a recent Drag Reduction Workshop ${ }^{2}$ organized by the American Institute of Aeronautics and Astronautics showed that drag was predicted by CFD to within an uncertainty of \pm 43 drag counts. This value is comparable to (although larger than) the uncertainty of \pm 8 drag counts extracted from results based on wind tunnel tests. This level of expectation from CFD data is far removed from that felt by CFD practitioners of high-speed reacting internal flows. Results presented at a recent Joint Army/Navy/NASA/Air Force Workshop on Turbulence and Kinetics Models for Scramjet Simulation included several examples where ReynoldsAveraged Navier-Stokes (RANS) models failed to even qualitatively mimic the fundamental flow physics present in these devices.

Higher order modeling approaches, such as Large Eddy Simulation (LES), offer significant advantages that overcome many of the shortcomings associated with the statistical representation of single-point RANS closures. The LES approach for turbulence closure attempts to resolve the large-scale components of turbulence while modeling the smaller scales. Most of the transport of mass, momentum, and energy (on the order of $90 \%$ ) is done by the large eddies, while the primary role of the small eddies is to dissipate these fluctuations. Hence, it is the large eddies that tend to interact directly with the mean flow. The resolution of the large scales implies that values chosen for modeled turbulent transport coefficients (e.g. turbulent Prandtl and Schmidt numbers) will have less of an impact on the overall flowfield prediction. The smaller turbulent scales tend to be isotropic in nature and less dependent on boundary conditions and flow type than the larger scales. Thus, the modeling developed for small scales should be more generally applicable than models developed for the entire range of turbulent scales. Unfortunately, the computational costs of LES often prohibit its use as an engineering design tool for practical applications. This is particularly true for attached wallbounded regions at modest to high Reynolds numbers. In fact, Spalart ${ }^{3}$ has estimated that the application of tradi- 
tional LES to an airliner wing would require on the order of $10^{20}$ floating point operations. This value is roughly one million times that of the largest RANS calculations attempted today.

The immense costs involved with resolving even a fraction of the turbulence spectra forces the continued reliance on single-point phenomenological models for the foreseeable future. Therefore, enhancements to Reynolds-Averaged Navier-Stokes methodologies will continue to be in high demand. This paper summarizes the current state-of-the-art modeling procedures used by engineers to model high-speed reacting flows. The typical set of equations used for high-speed propulsion applications are described along with all of the approximations (many of which are often taken for granted) required to close the averaged equation set. Some recent academic works meant to expand the applicability of the modeled equation set are also highlighted.

\section{GOVERNING EQUATIONS}

The equations that describe chemically reacting singlephase flows at conditions representative of most highspeed combustion applications are the Navier-Stokes equations coupled with $n s-1$ species mass continuity equations ( $n s$ is the number of species considered). These partial differential equations can be written as follows:

$$
\begin{aligned}
\frac{\partial \rho}{\partial t}+\frac{\partial}{\partial x_{j}}\left(\rho u_{j}\right) & =0 \\
\frac{\partial}{\partial t}\left(\rho u_{i}\right)+\frac{\partial}{\partial x_{j}}\left(\rho u_{i} u_{j}+\delta_{i j} P-\tau_{i j}\right) & =0 \\
\frac{\partial}{\partial t}(\rho E)+\frac{\partial}{\partial x_{j}}\left(\rho H u_{j}+q_{j}-\tau_{i j} u_{i}\right) & =0 \\
\frac{\partial}{\partial t}\left(\rho Y_{m}\right)+\frac{\partial}{\partial x_{j}}\left(\rho Y_{m} u_{j}+\rho Y_{m} V_{j}\right) & =\dot{w}_{m}
\end{aligned}
$$

where $\rho$ is the density, $u_{i}$ is the velocity, $P$ is the pressure, $E$ is the total energy, $H$ is the total enthalpy, $\tau_{i j}$ is the stress tensor, $q_{j}$ is the heat flux vector, and $Y_{m}, V_{j}$, and $\dot{w}_{m}$ are the mass fraction, diffusion velocity, and production rate, respectively, of species " $m$ ".

The time-averaged equations are obtained by decomposing each flow variable into a mean and fluctuating part. The following combination of conventional

$$
\phi=\bar{\phi}+\phi^{\prime}, \quad \bar{\phi} \equiv \lim _{\Delta t \rightarrow \infty} \frac{1}{\Delta t} \int_{t_{0}}^{t_{o}+\Delta t} \phi d t
$$

and mass-weighted

$$
\phi=\tilde{\phi}+\phi^{\prime \prime}, \quad \tilde{\phi} \equiv \frac{1}{\bar{\rho}} \lim _{\Delta t \rightarrow \infty} \frac{1}{\Delta t} \int_{t_{o}}^{t_{o}+\Delta t} \rho \phi d t
$$

decompositions,

$$
\begin{aligned}
\rho & =\bar{\rho}+\rho^{\prime} & u_{i} & =\tilde{u}_{i}+u_{i}^{\prime \prime} & P & =\bar{P}+P^{\prime} \\
\tau_{i j} & =\bar{\tau}_{i j}+\tau_{i j}^{\prime} & E & =\tilde{E}+E^{\prime \prime} & H & =\tilde{H}+H^{\prime \prime} \\
q_{i} & =\bar{q}_{i}+q_{i}^{\prime} & Y_{m} & =\tilde{Y}_{m}+Y_{m}^{\prime \prime} & \dot{w}_{m} & =\overline{\dot{w}}_{m}+\dot{w}_{m}^{\prime}
\end{aligned}
$$

is a common choice that minimizes the number of unknown correlations that appear. Substituting the decomposed variables (Eq. 4) into Eqs. 1a - 1d and averaging the result yields the desired time-averaged equation set:

$$
\begin{array}{r}
\frac{\partial \bar{\rho}}{\partial t}+\frac{\partial}{\partial x_{j}}\left(\bar{\rho} \tilde{u}_{j}\right)=0 \\
\frac{\partial}{\partial t}\left(\bar{\rho} \tilde{u}_{i}\right)+\frac{\partial}{\partial x_{j}}\left(\bar{\rho} \tilde{u}_{i} \tilde{u}_{j}+\delta_{i j} \bar{P}\right)= \\
\frac{\partial}{\partial x_{j}}\left(\bar{\tau}_{i j}-\bar{\rho} \widehat{u_{i}^{\prime \prime} u_{j}^{\prime \prime}}\right) \\
\frac{\partial}{\partial t}(\bar{\rho} \tilde{E})+\frac{\partial}{\partial x_{j}}\left(\bar{\rho} \tilde{H}_{\tilde{u}_{j}}\right)= \\
\frac{\partial}{\partial x_{j}}\left(\bar{\tau}_{i j} \tilde{u}_{i}+\bar{\tau}_{i j} u_{i}^{\prime \prime}-\bar{q}_{j}-\bar{\rho} \widehat{H^{\prime \prime} u_{j}^{\prime \prime}}\right) \\
\frac{\partial}{\partial t}\left(\bar{\rho} \tilde{Y}_{m}\right)+\frac{\partial}{\partial x_{j}}\left(\bar{\rho} \tilde{Y}_{m} \tilde{u}_{j}\right)= \\
\overline{\dot{w}}_{m}-\frac{\partial}{\partial x_{j}}\left(\widehat{\rho Y_{m} V_{j}}+\bar{\rho} \widehat{Y_{m}^{\prime \prime} u_{j}^{\prime \prime}}\right)
\end{array}
$$

All terms on the right-hand-side of Eqs. $5 b-5 d$ require modeling assumptions.

Two unclosed terms arise in the time-averaged momentum equation (Eq. 5b). The first term is the timeaveraged molecular stress tensor, and the second term is the Reynolds stress tensor. The Reynolds stress tensor is the predominant term, and nearly all of the modeling effort devoted towards the closure of the momentum equations has centered around this term. The classes of models available for this term will be described later. The remaining term $\left(\bar{\tau}_{i j}\right)$ has historically been modeled by ignoring the effects of turbulent fluctuations on the molecular viscosity, $\mu$, and assuming that the conventional $\left(\bar{u}_{i}\right)$ and mass-weighted $\left(\tilde{u}_{i}\right)$ average velocity are approximately equal. For a Newtonian fluid, these assumptions allow the average stress tensor to be approximated as follows:

$$
\begin{aligned}
\bar{\tau}_{i j} & =\mu \overline{\left(\frac{\partial u_{i}}{\partial x_{j}}+\frac{\partial u_{j}}{\partial x_{i}}\right)}-\frac{2}{3} \delta_{i j} \overline{\mu \frac{\partial u_{k}}{\partial x_{k}}} \\
& \approx \mu\left(\frac{\partial \tilde{u}_{i}}{\partial x_{j}}+\frac{\partial \tilde{u}_{j}}{\partial x_{i}}\right)-\frac{2}{3} \delta_{i j} \mu \frac{\partial \tilde{u}_{k}}{\partial x_{k}}
\end{aligned}
$$

Direct Numerical Simulation (DNS) studies ${ }^{4,5}$ have supported assumptions of this type, at least for perfect gases 
under mildly compressible conditions.

The time-averaged energy equation (Eq. $5 \mathrm{c}$ ) introduces three additional correlations that require modeling $\left(\bar{\tau}_{i j}\right.$ has already been considered). The first new term is a molecular diffusion term that is well approximated for incompressible flows by the following expression:

$$
\frac{\partial}{\partial x_{j}}\left(\overline{\tau_{i j} u_{i}^{\prime \prime}}\right) \approx \frac{\partial}{\partial x_{j}}\left(\mu \frac{\partial \tilde{k}}{\partial x_{j}}\right)
$$

where $\tilde{k}$ is the turbulent kinetic energy,

$$
\tilde{k}=\frac{1}{2} \widetilde{u_{i}^{\prime \prime} u_{i}^{\prime \prime}}
$$

For compressible flows, one typically assumes that this relationship remains valid. The time-averaged heat flux vector usually contains contributions from heat conduction and an energy flux due to inter-species diffusion, i.e.

$$
\bar{q}_{j}=-\overline{\frac{\partial T}{\partial x_{j}}}+\sum_{m=1}^{n s} \overline{\rho Y_{m} V_{j} h_{m}(T)}
$$

The contribution from heat conduction is modeled in a manner that is consistent with that done for the molecular stress tensor. That is, turbulent fluctuation effects are omitted when evaluating the thermal conductivity, $\lambda$, and the mass-weighted temperature fluctuation average, $\widetilde{T^{\prime \prime}}$, is assumed to be negligibly small, i.e.

$$
\lambda \overline{\frac{\partial T}{\partial x_{j}}} \approx \lambda \frac{\partial \tilde{T}}{\partial x_{j}}
$$

The treatment of the time-averaged inter-species diffusion term varies depending on the model chosen for the species diffusion velocity. The final term in Eq. $5 \mathrm{c}$ to be modeled is $\bar{\rho} \widetilde{H^{\prime \prime} u_{j}^{\prime \prime}}$. The average (mass-weighted) total enthalpy can be written in terms of the static enthalpy, $h$, and kinetic energy terms,

$$
\tilde{H}=\tilde{h}+\frac{1}{2}\left(\tilde{u}_{i} \tilde{u}_{i}+2 \tilde{k}\right)
$$

Subtracting this expression from the expanded instantaneous total enthalpy yields the fluctuating component of the total enthalpy, i.e.

$$
H^{\prime \prime}=h^{\prime \prime}+\tilde{u}_{i} u_{i}^{\prime \prime}+k^{\prime \prime}
$$

The unclosed correlation, $\bar{\rho} \widetilde{H^{\prime \prime} u_{j}^{\prime \prime}}$, can then be expanded to yield

$$
\bar{\rho} \widetilde{H^{\prime \prime} u_{j}^{\prime \prime}}=\bar{\rho} \widetilde{h^{\prime \prime} u_{j}^{\prime \prime}}+\bar{\rho} \tilde{u}_{i} \widetilde{u_{i}^{\prime \prime} u_{j}^{\prime \prime}}+\bar{\rho} \widehat{k^{\prime \prime} u_{j}^{\prime \prime}}
$$

The first term is the Reynolds heat flux vector. This term is modeled with various levels of complexity as will be shown later. The second term is the dot product of the mean velocity with the Reynolds stress tensor. This term is closed based on the model chosen for the Reynolds stress tensor. The third term represents turbulent transport of the turbulent kinetic energy. The gradient diffusion approximation is typically used to model this term,

$$
\bar{\rho} \widehat{k^{\prime \prime} u_{j}^{\prime \prime}}=-\frac{\mu_{t}}{\sigma_{k}} \frac{\partial \tilde{k}}{\partial x_{j}}
$$

where $\mu_{t}$ is the eddy viscosity and $\sigma_{k}$ is a closure coefficient defined by the chosen model for turbulence.

The remaining terms that require closure reside in the species continuity equations (Eq. 5d). The first term is the species production rate. A multitude of models exist for closing this term that range from simple eddy break-up / eddy dissipation models ${ }^{6,7}$ to more elaborate methods based on probability theory. ${ }^{8-10}$ A description of models that are typically used for high-speed reacting flows will be discussed later. The remaining unclosed terms are diffusion terms. The diffusion velocity of species " $m$ " is usually evaluated from Fick's law of diffusion, i.e.

$$
V_{j}=-\frac{D}{Y_{m}} \frac{\partial Y_{m}}{\partial x_{j}}
$$

when the Reynolds-averaged equation set is considered. In this expression, $D$ is the mass diffusivity of species " $\mathrm{m}$ " relative to the mixture. The use of this expression, in lieu of the costly evaluation of the multicomponent diffusion equation, is often justified by the premise that the "effective" turbulent diffusion is expected to dominate the molecular diffusion processes throughout most of the flowfield. Through Fick's law, the terms involving the species diffusion velocities are expressed as follows:

$$
\begin{aligned}
\overline{\rho Y_{m} V_{j}} & =-\overline{\rho D \frac{\partial Y_{m}}{\partial x_{j}}} \\
\sum_{m=1}^{n s} \overline{\rho Y_{m} V_{j} h_{m}(T)} & =-\sum_{m=1}^{n s} \overline{\rho D h_{m}(T) \frac{\partial Y_{m}}{\partial x_{j}}}
\end{aligned}
$$

If one employs the same approximations used to model the average molecular stress tensor (i.e. turbulent fluctuation effects neglected on the mixture diffusivity, and conventional averages assumed equivalent to mass-weighted averages), then these expression simplify to the following:

$$
\begin{aligned}
& \overline{\rho D \frac{\partial Y_{m}}{\partial x_{j}}} \approx \bar{\rho} D \frac{\partial \tilde{Y}_{m}}{\partial x_{j}} \\
& \sum_{m=1}^{n s} \overline{\rho D h_{m}(T) \frac{\partial Y_{m}}{\partial x_{j}}} \approx \sum_{m=1}^{n s} \bar{\rho} D h_{m}(\tilde{T}) \frac{\partial \tilde{Y}_{m}}{\partial x_{j}}
\end{aligned}
$$

Note that the effect of temperature fluctuations on the species enthalpy had to be ignored to arrive at the above 
expression for the averaged inter-species diffusion terms. This rather dubious approximation (along with the neglection of composition fluctuations) is also made when extracting the mean temperature from the conserved variables (or vice-versa). The final term to be modeled is the Reynolds mass flux vector, $\bar{\rho} \widehat{Y_{m}^{\prime \prime} u_{j}^{\prime \prime}}$. This term is usually modeled with the gradient diffusion approximation, although more complex models have also been used as will be shown later.

The model chosen for the equation of state introduces additional closure uncertainties. Even the simplest choice, where the fluid is assumed to behave as a mixture of perfect gases,

$$
P=\rho R T
$$

requires modeling assumptions since the gas "constant" $(R)$ varies with composition,

$$
R=R_{u} \sum_{m=1}^{n s} \frac{Y_{m}}{W_{m}}
$$

In this expression, $R_{u}$ is the universal gas constant and $W_{m}$ is the molecular weight of species " $m$ ". All efforts, known to the author, circumvent this closure difficulty by simply neglecting the effects of composition fluctuations on the equation of state, i.e.

$$
\bar{P}=\overline{\rho R T} \approx \tilde{\rho} R\left(\tilde{Y}_{m}\right) \tilde{T}
$$

\section{MODELING PRACTICES}

The previous section highlighted the numerous closure approximations that are required when modeling compressible reacting flows. This section discusses common closure approximations to the terms that are generally perceived to require the most attention by model developers. These terms are the Reynolds stress tensor $\left(\bar{\rho} \widetilde{u_{i}^{\prime \prime} u_{j}^{\prime \prime}}\right)$, Reynolds heat flux vector $\left(\bar{\rho} \widetilde{h^{\prime \prime} u_{j}^{\prime \prime}}\right)$, Reynolds mass flux vector $\left(\bar{\rho} \widetilde{Y_{m}^{\prime \prime} u_{j}^{\prime \prime}}\right)$, and the time-averaged chemical source term $\left(\overline{\dot{w}}_{m}\right)$. A description of models that are typically employed in high-speed combustion applications and their known deficiencies are described in the sections that follow.

\section{Reynolds Stress Tensor}

The most common closures used for the Reynolds stress tensor are linear models based on the Boussinesq approximation, i.e.

$$
\bar{\rho} \widetilde{u_{i}^{\prime \prime} u_{j}^{\prime \prime}}=\frac{2}{3} \delta_{i j}\left(\bar{\rho} \tilde{k}+\mu_{t} \frac{\partial \tilde{u}_{k}}{\partial x_{k}}\right)-\mu_{t}\left(\frac{\partial \tilde{u}_{i}}{\partial x_{j}}+\frac{\partial \tilde{u}_{j}}{\partial x_{i}}\right)
$$

These models assume that the Reynolds stress components are related to the mean strain rate tensor through an isotropic eddy viscosity $\left(\mu_{t}\right)$. Models for the eddy viscosity vary in complexity from simple algebraic (zeroequation) models ${ }^{11}$ which require specification of a turbulent velocity and length scale, to two-equation models $12-15$ which solve partial differential equations for both the turbulent velocity scale and an additional turbulence scale (e.g. a length scale, time scale, or dissipation rate). A three-equation $\left(k-\varepsilon-\overline{v^{2}}\right)$ model has also been proposed in the literature, ${ }^{16}$ although this model has not been extensively applied to high-speed reacting flows. Algebraic models have the advantage of being numerically robust and easy to implement (at least for relatively simple geometries). However, these models often require changes in their coefficients when applied to different types of flowfields, and ambiguities often arise when defining the turbulence scales for complex geometries. Two-equation models, on the other hand, tend to have a larger range of applicability, and they are easily extended to complex geometries where it may be difficult to define relevant turbulent scales algebraically. Oneequation models, that involve a transport equation for a quantity that can be directly related to the eddy viscosity, ${ }^{17,18}$ have gained popularity in recent years, particularly for external flow applications. This trend has not yet been seen, to a large degree, for internal reacting flows.

The linear eddy viscosity models described previously have several deficiencies that are rectified by invoking higher order models. The first deficiency is a result of the direct proportionality assumed between the Reynolds stress and mean strain rate tensors (i.e. the Boussinesq approximation). This feature prevents the prediction of secondary flow motions that result from Reynolds stress anisotropies. Moreover, these models do not incorporate the influences of pressure-strain correlations, which are responsible for the distribution of anisotropy among the normal stress components. The linear eddy viscosity models are also unable to rigorously account for streamline curvature effects, since the Reynolds stresses depend solely on the frame-invariant strain rate tensor. These deficiencies are resolved through the use of second order models that involve transport equations for each of the Reynolds stress components, i.e.

$$
\begin{aligned}
& \frac{\partial}{\partial t}\left(\bar{\rho} \widetilde{u_{i}^{\prime \prime} u_{j}^{\prime \prime}}\right)+\underbrace{\frac{\partial}{\partial x_{k}}\left(\bar{\rho} \widetilde{u_{i}^{\prime \prime} u_{j}^{\prime \prime}} \tilde{u}_{k}\right)}=-\underbrace{\frac{\partial}{\partial x_{k}}\left(\bar{\rho} u_{i}^{\prime \prime} u_{j}^{\prime \prime} u_{k}^{\prime \prime}\right)} \\
& \text { (I) } \\
& \text { (II) }
\end{aligned}
$$

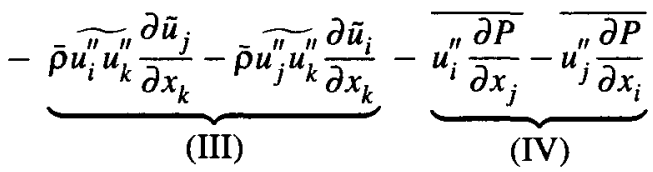

$$
\begin{aligned}
& +\underbrace{\overline{u_{i}^{\prime \prime} \frac{\partial \tau_{j k}}{\partial x_{k}}+\overline{u_{j}^{\prime \prime} \frac{\partial \tau_{i k}}{\partial x_{k}}}}}_{(\mathrm{V})}
\end{aligned}
$$


This expression shows that the Reynolds stress tensor is influenced by: (I) advection, (II) turbulent convection, (III) source/sink due to the mean velocity gradient, (IV) source/sink due to the pressure gradient, and (V) dissipation due to viscosity. The unclosed terms are terms (II), (IV), and (V). The chain rule has historically been applied to term (IV), resulting in an additional diffusion term and a pressure-strain rate correlation,

$$
\begin{aligned}
\overline{u_{i}^{\prime \prime} \frac{\partial P}{\partial x_{j}}}+\overline{u_{j}^{\prime \prime} \frac{\partial P}{\partial x_{i}}} & =\frac{\partial}{\partial x_{k}}\left[\overline{P u_{i}^{\prime \prime}} \delta_{j k}+\overline{P u_{j}^{\prime \prime}} \delta_{i k}\right] \\
& -\left[\overline{P \frac{\partial u_{i}^{\prime \prime}}{\partial x_{j}}}+\overline{P \frac{\partial u_{j}^{\prime \prime}}{\partial x_{i}}}\right]
\end{aligned}
$$

Similar expansions are applied to term (V) to yield a diffusion term and a dissipation term, i.e.

$$
\begin{aligned}
\overline{u_{i}^{\prime \prime} \frac{\partial \tau_{j k}}{\partial x_{k}}}+\overline{u_{j}^{\prime \prime} \frac{\partial \tau_{i k}}{\partial x_{k}}} & =\frac{\partial}{\partial x_{k}}\left[\overline{u_{i}^{\prime \prime} \tau_{j k}}+\overline{u_{j}^{\prime \prime} \tau_{i k}}\right] \\
& -\left[\overline{\tau_{j k} \frac{\partial u_{i}^{\prime \prime}}{\partial x_{k}}}+\overline{\tau_{i k} \frac{\partial u_{j}^{\prime \prime}}{\partial x_{k}}}\right]
\end{aligned}
$$

The pressure-strain rate correlation is responsible for the distribution of anisotropy among the normal stress components. This term is often of the same order of magnitude as the source term due to the mean velocity gradient, term (III). Hence, substantial efforts have been devoted towards the modeling of this term. This term is usually partitioned into a "slow" relaxation towards isotropy term and a "rapid" response term resulting from imposed mean velocity gradients. ${ }^{19-21}$ The dissipation term in Eq. 24 is typically partitioned into isotropic and deviatoric components, with the deviatoric component neglected in most works, i.e.

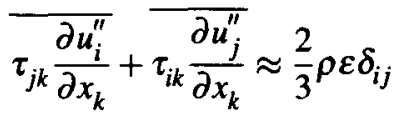

where

$$
\varepsilon=\overline{\frac{\mu}{\rho} \frac{\partial u_{i}^{\prime}}{\partial x_{k}} \frac{\partial u_{i}^{\prime}}{\partial x_{k}}}
$$

Models for the third order velocity correlation, term (II), can be found in Refs. 22 - 24. This term accounts for the turbulent convection of the Reynolds stress and is modeled to mimic a diffusion process.

The computational cost associated with solving the Reynolds stress transport equations has discouraged its use for complex engineering calculations. The increased cost is due to the additional transport equations, and the stiffness posed by the highly non-linear relationships introduced to close these equations. This has led many to consider algebraic closures derived from the Reynolds stress equation. Implicit algebraic models ${ }^{25}$ are obtained by enforcing equilibrium assumptions on the turbulence. The specific assumptions are that the turbulence has reached an equilibrium state, i.e.

$$
\frac{D}{D t}\left(\bar{\rho} \widetilde{u_{i}^{\prime \prime} u_{j}^{\prime \prime}}\right)=\left[\frac{\bar{\rho} \widetilde{u_{i}^{\prime \prime} u_{j}^{\prime \prime}}}{\tilde{k}}\right] \frac{D \tilde{k}}{D t}
$$

and any anisotropies resulting from the turbulent transport and diffusion terms are proportional to anisotropies in the Reynolds stresses,

$$
\begin{aligned}
& \frac{\partial}{\partial x_{k}}\left(\bar{\rho} u_{i}^{\prime \prime u_{j}^{\prime \prime} u_{k}^{\prime \prime}}+\overline{P u_{i}^{\prime \prime}} \delta_{j k}+\widehat{P u_{j}^{\prime \prime}} \delta_{i k}-\overline{u_{i}^{\prime \prime} \tau_{j k}}-\overline{u_{j}^{\prime \prime} \tau_{i k}}\right)= \\
& {\left[\frac{\bar{\rho} \widetilde{u_{i}^{\prime \prime} u_{j}^{\prime \prime}}}{\tilde{k}}\right] \frac{\partial}{\partial x_{k}}\left(\bar{\rho} \widetilde{k u_{k}^{\prime \prime}}+\overline{P u_{k}^{\prime \prime}}-\overline{\tau_{i k} u_{i}^{\prime \prime}}\right)}
\end{aligned}
$$

Unfortunately, the iterative process required to solve the resulting system of equations has proven to be extremely "stiff". Pope ${ }^{26}$ was able to cast these expressions into a set of equations that result in explicit relationships for the Reynolds stresses for two-dimensional flows. This approach was subsequently extended to three-dimensional flows. ${ }^{27}, 28$ Algebraic Reynolds stress models, in contrast to linear eddy viscosity models, retain the information from the pressure-strain correlation models of the full Reynolds stress closure, and allow for Reynolds stress anisotropies. Of course, the applicability of the formulation hinges on the validity of the equilibrium assumptions given by Eqs. 27 and 28 . The computational expense associated with explicit algebraic Reynolds stress models (EASM) is only slightly greater than that required for standard two-equation variants of linear eddy viscosity closures.

The importance of accounting for Reynolds stress anisotropies can be illustrated by considering flow through a rectangular duct. These flowfields are known to contain stress-induced secondary motions near the corners of the duct, which develop due to Reynolds stress anisotropies. Computed pitot pressure distributions extracted from simulations of a Mach 3.9 flow in a square duct are compared with measurements ${ }^{29}$ in Figs. and . In these figures, the measured data is shown on the left of the symmetry plane, while the computed results are shown on the right. Fig. compares computed results using the linear Wilcox $k-\omega$ model $^{13}$ to measurements, while Fig. compares computed results using an explicit algebraic Reynolds stress model ${ }^{30}$ to measurements. When the Reynolds stress anisotropies are not accounted for (Fig. ), secondary flow structures do not develop in the corner region of the duct. As a result, the boundary layer builds up more rapidly near the corners. The algebraic Reynolds stress model accounts for the stress anisotropies, allow- 
ing the secondary flow structures to develop. These structures transport high momentum fluid from the core flow into the corner regions which results in a "squared-off" boundary layer profile that more closely matches the experimental data. The importance of accounting for the secondary flow structures is further illustrated in the wall pressure trace along the duct (see Fig. ). The boundary layer growth is over-predicted in the linear $k-\omega$ results, yielding a larger total pressure loss in the latter half of the duct. Rectangular flowpaths are prevalent in many scramjet propulsion systems, hence accounting for the stress anisotropies may prove to be a critical ingredient when assessing inlet and isolator performance.

As a final note, a few statements should be made for the class of models known simply as non-linear eddy viscosity models (NLEVM). ${ }^{31-33}$ These models are functionally similar to EASM models, in the sense that the Reynolds stress tensor is represented by a polynomial expansion of some given tensorial basis. ${ }^{34}$ The primary difference between EASM and NLEVM models is the manner in which the expansion coefficients are determined. The expansion coefficients derived for EASM models are based on the mathematical procedure followed to recast the implicit Reynolds stress expressions into explicit relationships. The expansion coefficients determined for NLEVM models, on the other hand, are based on empiricism and realizability constraints.

\section{Reynolds Heat/Mass Flux Vector}

The turbulent transport of a scalar property has historically been modeled using the gradient diffusion hypothesis. This model choice assumes that the turbulent transport of the scalar is in the direction of decreasing value for that scalar. This leads to the following model expressions for the Reynolds heat flux

$$
\bar{\rho} \widetilde{h^{\prime \prime} u_{j}^{\prime \prime}}=-\frac{\mu_{t}}{P r_{t}} \frac{\partial \tilde{h}}{\partial x_{j}}
$$

and mass flux

$$
\widetilde{\rho} \widetilde{Y_{m}^{\prime \prime} u_{j}^{\prime \prime}}=-\frac{\mu_{t}}{S c_{t}} \frac{\partial \tilde{Y}_{m}}{\partial x_{j}}
$$

vectors. The diffusion rates are controlled by specifying the turbulent Prandtl $\left(\operatorname{Pr}_{t}\right)$ and Schmidt $\left(S c_{t}\right)$ numbers. The turbulent Prandtl number specifies the ratio of the rate of turbulent momentum transport to rate of turbulent energy transport, while the turbulent Schmidt number defines the ratio of the turbulent momentum transport rate to turbulent mass transport rate. Constant values for these coefficients are usually assumed in applications for low- and high-speed reacting flows of engineering interest, even though values for these coefficients have been shown to vary spatially. ${ }^{35-45}$ Table 1 summarizes the range of values that have been observed (both experimentally and computationally) for various flows.

Calculations performed by this author ${ }^{46,47}$ and other works ${ }^{43,48}$ have at times shown an extreme sensitivity to values assumed for these parameters. An example is taken from Ref. 47 involving calculations performed for a direct connect scramjet combustor (see Fig.) tested at Wright-Patterson Air Force Base (AFRL/PRA). Figures and show mass-flux weighted flow properties through the combustor at flight conditions that correspond to Mach 4.0 and Mach 6.5 operation. Results are shown for various $P r_{t}$ values with $S c_{t}$ fixed at 0.5 , and for several $S c_{t}$ values with $P r_{t}$ fixed at 0.89 . The range of values considered is within the range of values given in Table 1. As one would expect, reducing the turbulent Schmidt number consistently intensified combustion due to enhanced species diffusion processes. At the Mach 4.0 condition, the reduction of $S c_{t}$ from 0.5 to 0.25 enhanced turbulent mass transfer (and subsequent heat release) to levels that the isolator was not able to withstand, resulting in a potentially catastrophic un-start condition. A modest increase of $S c_{t}$ from 0.5 to 0.75 reduced the turbulent mass transfer to levels that were not able to sustain combustion. Hence, a variation in $S c_{t}$ from 0.25 to 0.75 yielded results that covered the entire spectrum of operability for the engine at the Mach 4.0 flight condition. $A$ reduction of the turbulent Prandtl number enhanced the combustion process only at the higher Mach number state. At the Mach 4.0 condition, the heightened thermal diffusion processes allowed heat to be transferred away from the flameholding (recirculation) zones at a rate that was not sustainable, causing flame blow-out. These results clearly suggest that extreme care should be taken when attempting to characterize these high-speed propulsion devices (that contain a variety of different mixing mechanisms) with constant turbulent transport coefficients.

Table 1: Turbulent Prandtl \& Schmidt Number Values

\begin{tabular}{||c|c|c||}
\hline \hline Flow Field & $P r_{t}$ & $S c_{t}$ \\
\hline \hline Planar Jets ${ }^{35-38}$ & $0.2-3.0$ & $0.1-2.2$ \\
Round Jets $^{39-41}$ & $0.7-2.0$ & $0.1-2.0$ \\
Backward Facing Step $^{42}$ & $0.7-3.0$ & NA \\
Jet into Cross Flow 43,44 & NA & $0.1-0.5$ \\
Injection Behind a Bluff Body ${ }^{45}$ & NA & $0.2-0.7$ \\
\hline \hline
\end{tabular}

The physical mechanisms that directly influence the Reynolds heat and mass flux vectors can be ascertained by examining the transport equations for these quantities. The transport equations that govern the Reynolds 
heat and mass flux vectors can be written as:

$$
\frac{\partial}{\partial t}\left(\bar{\rho} \widetilde{h^{\prime \prime} u_{j}^{\prime \prime}}\right)+\underbrace{\frac{\partial}{\partial x_{i}}\left(\bar{\rho} \widetilde{h^{\prime \prime} u_{j}^{\prime \prime}} \tilde{u}_{i}\right)}_{(\bar{\rho})}=-\underbrace{\frac{\partial}{\partial x_{i}}\left(\bar{\rho} u_{i}^{\prime \prime} u_{j}^{\prime \prime} h^{\prime \prime}\right)}
$$

(II)

$$
\begin{aligned}
& -\underbrace{\bar{\rho} \widehat{h^{\prime \prime} u_{i}^{\prime \prime}} \frac{\partial \tilde{u}_{j}}{\partial x_{i}}}_{(\mathrm{III})}-\underbrace{\bar{\rho} \widetilde{u_{i}^{\prime \prime} u_{j}^{\prime \prime} \frac{\partial \tilde{h}}{\partial x_{i}}}}_{(\mathrm{IV})}-\underbrace{\overline{h^{\prime \prime} \frac{\partial P}{\partial x_{j}}}}_{\text {(V) }}+\underbrace{\overline{h^{\prime \prime} \frac{\partial \tau_{i j}}{\partial x_{i}}}}_{\text {(VI) }} \\
& -\underbrace{\overline{u_{j}^{\prime \prime} \frac{\partial q_{i}}{\partial x_{i}}}}_{\text {(VII) }}+\underbrace{\overline{u_{j}^{\prime \prime} \tau_{i k} \frac{\partial u_{k}}{\partial x_{i}}}+\overline{u_{j}^{\prime \prime} \frac{D P}{D t}}}_{\text {(VIII) }}
\end{aligned}
$$

and

$$
\begin{aligned}
& \frac{\partial}{\partial t}\left(\bar{\rho} \widetilde{Y_{m}^{\prime \prime} u_{j}^{\prime \prime}}\right)+\underbrace{\frac{\partial}{\partial x_{i}}\left(\bar{\rho} \widetilde{Y_{m}^{\prime \prime} u_{j}^{\prime \prime}} \tilde{u}_{i}\right)}=-\underbrace{\frac{\partial}{\partial x_{i}}\left(\bar{\rho} u_{i}^{\prime \prime} u_{j}^{\prime \prime} Y_{m}^{\prime \prime}\right)} \\
& \text { (I) } \\
& \text { (II) } \\
& -\underbrace{\bar{\rho} \widetilde{Y_{m}^{\prime \prime} u_{i}^{\prime \prime} \frac{\partial \tilde{u}_{j}}{\partial x_{i}}}}_{\text {(III) }}-\underbrace{\bar{\rho} \widetilde{u_{i}^{\prime \prime} u_{j}^{\prime \prime} \frac{\partial \tilde{Y}_{m}}{\partial x_{i}}}}_{(\mathrm{IV})}-\underbrace{\overline{Y_{m}^{\prime \prime} \frac{\partial P}{\partial x_{j}}}}_{(\mathrm{V})}+\underbrace{\overline{Y_{m}^{\prime \prime} \frac{\partial \tau_{i j}}{\partial x_{i}}}}_{\text {(VI) }} \\
& +\underbrace{\overline{u_{j}^{\prime \prime} \frac{\partial}{\partial x_{i}}\left(\rho D \frac{\partial Y_{m}}{\partial x_{i}}\right)}}_{(\mathrm{VII})}+\underbrace{\overline{u_{j}^{\prime \prime} \dot{w}_{m}}}_{(\mathrm{VIII})}
\end{aligned}
$$

The above expressions show that the evolution of each Reynolds flux vector is governed by: (I) advection, (II) turbulent convection, (III) source/sink due to the mean velocity gradient, (IV) source/sink due to the mean scalar gradient, (V) source/sink due to the pressure gradient, (VI) dissipation due to viscosity, and (VII) dissipation due to scalar diffusivity. Note that the Reynolds mass flux vector is also directly affected by the chemistry (VIII). Clearly, any attempt to collapse all of these physical phenomena into a single gradient diffusion effect is questionable. In fact, the literature is filled with evidence of counter-gradient diffusion effects ${ }^{49-51}$ (i.e. turbulent diffusion of a scalar against its mean gradient) on the Reynolds flux vectors, particularly in pre-mixed applications. Counter-gradient diffusion has been attributed to the mean pressure gradient portion of term $(\mathrm{V})$ in Eqs. 31 and 32.

The number of scalar transport expressions that result from Eqs. 31 and 32 is $3 \times n s$. Additional supporting turbulent transport equations for variances/covariances and their dissipation rates are also typically required to model the unclosed terms on the right-hand-side of these equations. Hence, even if suitable models were developed to close each of the scalar flux vector equations, the number of additional equations introduced would greatly exceed the equation count given by the first order moments of
Eqs. 5a - 5b. This fact suggests that it would be impractical to include a full second order closure model in any simulation of engineering interest. To circumvent this difficulty, some limiting studies have invoked equilibrium assumptions to reduce the differential equations to algebraic relationships. Other studies have coupled the gradient diffusion hypothesis with models that allow the turbulent Prandtl and/or Schmidt number to vary spatially.

The work of Adumitroaie ${ }^{52}$ involved the development and application of a complete algebraic closure for the Reynolds stress tensor and scalar flux vectors. The explicit algebraic Reynolds stress model used in this effort was based on the closure of Taulbee ${ }^{27}$ and included compressibility effects. The algebraic Reynolds scalar flux models for temperature and species composition were derived in Ref. 52 based on similar principles. The model neglected scalar correlations higher than second order and cross-correlations between temperature and composition were neglected (as were temperature fluctuation effects on the reaction rates). Additional transport equations (beyond those given by Eqs. $5 \mathrm{a}-5 \mathrm{~b}$ ) required by the model include the turbulent kinetic energy and its dissipation rate, the variance of temperature and its dissipation rate, and the variances and covariances of the $n s-1$ composition variables and their dissipation rates. The end result is that $n s \times(n s-1)+4$ additional transport equations are introduced; a value that exceeds the equation count for the first order moments for $n s>2$. Nevertheless, encouraging results were obtained for a compressible mixing layer (cold flow) and planar jet when compared with results obtained from a fully second order moment transport model. The author noted that high shear regions were problematic with the model, suggesting that the highly nonlinear nature of the algebraic closures could pose difficulties in complex flows. Further numerical difficulties associated with the use of the algebraic Reynolds mass flux expressions were noted by the author when chemical reactions were considered.

The development of models that allow for variable turbulent Prandtl/Schmidt numbers within the context of the gradient diffusion hypothesis has been pursued by several authors. ${ }^{40,41,44}$ The variable turbulent Prandtl number models tend to involve additional transport equations for the temperature or enthalpy/energy variance $\left(g^{\prime \prime} g^{\prime \prime}\right)$ and its dissipation rate $\left(\varepsilon_{g}\right)$. This allows an additional (independent) turbulent time scale to be introduced into the definition of the thermal eddy diffusivity:

$$
\alpha_{t} \equiv \frac{\mu_{t}}{P r_{t}}=C_{T} \tilde{k} \tau_{T}
$$

where $C_{T}$ is a model coefficient (possibly with a nearwall damping function) and $\tau_{T}$ is some measure of the turbulent thermal time scale. The thermal time scale can be based purely on the scalar transport variables, or a 
mixed time scale can be defined by introducing the turbulent time scale based on the velocity field $(\tau)$, i.e.

$$
\tau_{T}=\left(\frac{\widetilde{g^{\prime \prime} g^{\prime \prime}}}{\varepsilon_{g}}\right) \quad \text { or } \quad \tau_{T}=\sqrt{\tau}\left(\frac{\widetilde{g^{\prime \prime} g^{\prime \prime}}}{\varepsilon_{g}}\right)^{\frac{1}{2}}
$$

These particular choices for the thermal time scale yield the following expressions for the turbulent Prandtl number:

$$
\operatorname{Pr}_{t}=\frac{\mu_{t}}{C_{T^{\tilde{k}}}}\left(\frac{\varepsilon_{g}}{\widehat{g^{\prime \prime} g^{\prime \prime}}}\right) \quad \text { or } \quad \operatorname{Pr}_{t}=\frac{\mu_{t}}{C_{T} \tilde{k} \sqrt{\tau}}\left(\frac{\varepsilon_{g}}{\widehat{g^{\prime \prime} g^{\prime \prime}}}\right)^{\frac{1}{2}}
$$

Variable turbulent Schmidt number models are arrived at in a similar fashion by integrating evolution equations for some measure of the composition variance (e.g. the mixture fraction variance or the sum of all species mass fraction variances) and its dissipation rate. If these quantities are also denoted by $\widetilde{g^{\prime \prime} g^{\prime \prime}}$ and $\varepsilon_{g}$, then the expressions related to the eddy diffusivity of mass are obtained from Eqs. 33, 34 , and 35 by replacing $\operatorname{Pr}_{t}, C_{T}$, and $\tau_{T}$ with $S c_{t}, C_{M}$, and $\tau_{M}$.

\section{Chemical Production Rate}

The most common species production rate closures used for high-speed reacting flows are based on laminarchemistry, eddy break-up / dissipation, ${ }^{6,7}$ or probability density function (PDF) ${ }^{8-10}$ formulations. Approaches based on a laminar-chemistry assumption simply ignore turbulence-chemistry interactions by evaluating the chemical source terms based on mean flow properties. Eddy break-up models are mixing limited models where the chemical time scale is assumed to be limited by the dissipation rate of turbulent eddies. Formulations based on ideas borrowed from probability theory represent perhaps the most elegant class of models for averaging the chemical source terms. However, these formulations can be considerably more expensive to invoke.

Let a general kinetic step be denoted as follows:

$$
\sum_{m=1}^{n s+1} v_{m l}^{\prime} C_{m} \rightleftharpoons \sum_{m=1}^{n s+1} v_{m l}^{\prime \prime} C_{m} \quad l=1, n r
$$

where $v_{m l}^{\prime}$ and $v_{m l}^{\prime \prime}$ are the reactant and product stoichiometric coefficients for species " $m$ " in reaction " $l$ ", $C_{m}$ is the symbol for constituent " $m$ " (the $n s+1$ constituent representing the third body species), and $n r$ is the number of chemical reactions considered. The expressions used for the chemical source terms are then generally given by the law of mass action or empirically derived global reaction rate expressions. The law of mass action applies to reaction models that are based on elementary kinetic steps, and can be written as follows:

$$
\begin{aligned}
\dot{w}_{m}= & W_{m} \sum_{l=1}^{n r}\left(v_{m l}^{\prime \prime}-v_{m l}^{\prime}\right) \\
& {\left[k_{f_{l}} \prod_{n=1}^{n s+1}\left(\frac{\rho_{n}}{W_{n}}\right)^{v_{n l}^{\prime}}-k_{b_{l}} \prod_{n=1}^{n s+1}\left(\frac{\rho_{n}}{W_{n}}\right)^{v_{n l}^{\prime \prime}}\right] }
\end{aligned}
$$

where $k_{f_{l}}$ and $k_{b_{l}}$ are the forward and backward reaction rate coefficients of reaction "l" (typically exponential functions of temperature), and $\rho_{n}$ is the density of species " $n$ ". The molar concentration of the third body constituent in Eq. 37 is defined by the following expression:

$$
\frac{\rho_{n s+1}}{W_{n s+1}}=\sum_{m=1}^{n s} t b e_{m l} \frac{\rho_{m}}{W_{m}}
$$

where $t b e_{m l}$ is the third body efficiency of species " $\mathrm{m}$ " in reaction "l" provided with the kinetic model. The chemical source term based on one-way global steps (sometimes referred to as arbitrary reaction order steps) can be written in the following manner:

$$
\dot{w}_{m}=W_{m} \sum_{l=1}^{n r}\left(v_{m l}^{\prime \prime}-v_{m l}^{\prime}\right) k_{f_{l}} \prod_{n=1}^{n s}\left(\frac{\rho_{n}}{W_{n}}\right)^{a_{n l}}
$$

Here, the coefficient $a_{n l}$, in general, is not equal to the stoichiometric coefficient of species " $n$ " in reaction " $l$ " as is the case with the law of mass action. This coefficient is instead determined empirically using data generated from measurements or from a detailed kinetic mechanism.

The species production rates are point functions (i.e. functions that are defined by variables at a single spatial and temporal location), thus they are ideally suited for single point PDF closures. The source terms, given by Eqs. 37 and 39, are a function of temperature and composition only. As a result, these terms can be averaged by integrating the product of the species production rates with the joint PDF $(\mathscr{P})$ of temperature and composition at each spatial location, i.e.

$$
\begin{aligned}
\bar{w}_{m}= & \int \dot{w}_{m}\left(\hat{T}, \hat{\rho}_{1}, \ldots, \hat{\rho}_{n s}\right) \mathscr{P}\left(\hat{T}, \hat{\rho}_{1}, \ldots, \hat{\rho}_{n s}\right) \\
& d \hat{T} d \hat{\rho}_{1} \ldots d \hat{\rho}_{n s}
\end{aligned}
$$

The integration in the above expression is taken over all realizable values for temperature and composition, and the independent variables of the PDF ( $\hat{T}$ and $\hat{\rho}_{m}$ ) represent the sample space of the random variables $T$ and $\rho_{m}$. The form for the joint PDF can be assumed a priori ${ }^{53-57}$ or by integrating the evolution equation governing the PDF. ${ }^{8-10}$ Note that assumed PDF formulations based on mixture fraction, ${ }^{53}$ which are popular in low-speed applications, are seldom used in high-speed flows. These approaches tend to treat the reacting system as either a mixed-is-burned flame sheet or assume the mixture is in 
chemical equilibrium. Neither infinitely fast chemistry assumption is appropriate in supersonic flows due to limited flow residence times.

The computational overhead associated with invoking a PDF approach varies greatly depending on the particular formulation invoked. Assumed PDF methods typically add $10-30 \%$ overhead over a laminar-chemistry calculation, provided that the integrations in Eq. 40 can be performed analytically or through an efficient table lookup procedure. Approaches that involve the integration of an evolution equation governing the joint PDF are considerably more expensive, possibly by as much as a factor of 10 or more over their laminar-chemistry counter-parts. Due to the large dimensionality of the joint PDF, solving the evolution equation with a finite difference scheme is not practical. ${ }^{10}$ Instead, the equation is typically simulated using a Monte Carlo scheme. The additional computational cost is then dependent on the number of representative sample space ensembles used for the Monte Carlo simulation.

Calculations of high-speed reacting flows that have accounted for turbulence-chemistry interactions through the use of PDF formulations can be found in Refs. 46, 58 61. One observation found from each of these sources is that the effect of turbulence-chemistry interactions is relatively minor except in the vicinity of flame ignition. Figure compares results obtained for a supersonic axisymmetric $\mathrm{H}_{2}$ /Air burner ${ }^{62}$ using laminar-chemistry, assumed PDF, and evolution PDF closure approximations. The assumed PDF model invoked a Gaussian distribution for temperature fluctuations and a multi-variate $\beta$ distribution ${ }^{63}$ for composition fluctuations. Reaction crosscorrelations (RCC) ${ }^{61}$ between temperature and composition were neglected in the model. The evolved PDF results were obtained by integrating an equation governing the scalar probability density function for enthalpy and composition via a Eulerian Monte Carlo procedure. ${ }^{10}$

The similarity observed in the results extracted from each turbulence-chemistry closure is an outcome that is contrary to what is typically expected in low-speed applications. Large scale mixing within turbulent eddies tends to "stir" the fuel and air streams rather than mix them at a scale small enough for chemical reactions to take place. Hence, the average mass fractions within a control volume larger than the eddy would suggest that the two streams are well mixed, but in reality the two stream may still be segregated within the eddy. This phenomenon, referred to in the literature as unmixedness, leads to large scalar covariance levels, and tends to substantially reduce the magnitude of the species production rate as compared with results based on a laminar-chemistry treatment. One explanation as to why this phenomenon is not as prevalent in high-speed flows was described in Ref. 38. In this work, LES was used to examine the scalar fluctua- tion statistics of low-speed and high-speed mixing layers. This effort showed that the mixture fraction variance extracted from the high-speed compressible mixing layer was significantly lower than the values extracted from the low-speed case. Moreover, a measure of the mixed fluid probability and the peak mean temperature were both higher for the high-speed mixing layer. These observations suggest that the concept of unmixedness may be play a smaller role in high-speed reacting flows.

Models based on the eddy dissipation concept address the turbulence closure problem by assuming that the kinetic rate is limited by the rate of mixing (on a molecular scale) between fuel and oxygen carrying eddies rather than on the chemical time scale. In regions of high turbulence levels, the eddy lifetime is short leading to large eddy dissipation rates and more rapid molecular mixing than regions of lower turbulence levels. This model is applicable to irreversible reactions only, and is usually applied to a single reaction step such as:

$$
\text { Fuel }+v_{A} \text { Air } \rightarrow v_{P} \text { Products }
$$

where the stoichiometric coefficients $\left(v_{A}, v_{P}\right)$ are related to the stoichiometric Air to Fuel mass ratio $(A / F)$, i.e.

$$
v_{A}=\frac{W_{F}}{W_{A}}\left(\frac{A}{F}\right)_{s t}, \quad v_{P}=\frac{W_{F}}{W_{P}}\left[\left(\frac{A}{F}\right)_{s t}+1\right]
$$

The chemical source term based on the eddy dissipation concept proposed by Magnussen and Hjertager ${ }^{7}$ is given by the following relationship:

$$
\dot{w}_{m}=W_{m}\left(v_{m}^{\prime \prime}-v_{m}^{\prime}\right) \frac{A}{\tau} \mathrm{MIN}\left[\frac{\rho_{F}}{W_{F}}, \frac{\rho_{A}}{v_{A} W_{A}}, B \frac{\rho_{P}}{v_{P} W_{P}}\right]
$$

where $A$ and $B$ are empirical constants originally set to 4.0 and 0.5 , respectively in Ref. 7. This model is popular due to its simplicity and its dependence only on first order correlations (i.e. no additional transport equations are required). Many implementations of this model also permit the chemical time scale to be considered as a limiting rate using either Eq. 37 or 39 . In this scenario, the expression that yields the smallest magnitude for the reaction rate is the expression used to compute the source term. This additional limit discourages chemical reactions in cold regions of the flowfield. One clear advantage of the pure model given by Eq. 43 is that reaction rate coefficients, which are often not available for complex fuels, are not required. This model also requires a minimal number of species transport equations, making it computationally efficient. Moreover, the use of a single time scale for reaction $(\tau)$ alleviates much of the stiffness involved with more complex chemical systems. The major drawback of this model is that the details of the chemical processes are neglected. Consequently, models 
of this type should never be used to predict lean blow-out limits or combustor ignition characteristics.

\section{CONCLUSIONS}

The use of steady-state RANS models has been and will continue to be the tool of choice for modeling compressible reacting flows for high-speed commercial and military applications. Even with expected increases in computer speed, the role of LES will likely be limited to idealized component analysis, or to scenarios where flow unsteadiness is of special concern. Hence, improvements to modeling approaches for compressible reacting flows within a RANS framework offers the greatest potential advancement to CFD practitioners. Of the issues raised in this document, improvements to the turbulent scalar transport models are likely to reap the most benefits. The simple gradient diffusion models with constant transport coefficients have proven to be particularly troublesome. When one considers the limited residence times associated with scramjet engine flows (typically on the order of one millisecond), it is not surprising how even a small discrepancy in mixing rate prediction can lead to large deviations in combustor performance. Considerably more attention has historically been given to higher order models for closure of the Reynolds stress tensor (at least in lowspeed applications). The use of models from this class is envisioned to improve upon predictions of hypersonic inlet and isolator flows which are dominated by shockinduced separation and Reynolds stress anisotropies. Linear Reynolds stress models are certainly not capable of predicting the latter of these flow scenarios. Calculations to date have yet to show a first order need for the advancement of turbulence-chemistry interaction models in high-speed applications; although this issue has not yet been thoroughly addressed. This observation is in stark contrast to low-speed reacting flows where these models are required to avoid a significant over-prediction of the mean temperature field. At this time, imprecise results associated with the modeling of turbulence-chemistry interactions tend to be overshadowed by inaccuracies in turbulent scalar transport predictions.

\section{REFERENCES}

[1] van Dam, C. P. "Critical Factors in CFD-Based Drag Prediction". VKI Lecture Series, von Karman Institute for Fluid Dynamics, Nov. 2003.

[2] Hemsch, M. "Statistical Analysis of CFD Solutions from the Drag Prediction Workshop". AIAA Paper 2002-0842, Jan. 2002.

[3] Spalart, P. R., Jou, W.-H., Strelets, M., and Allmaras, S. R. "Comments on the Feasibility of LES for Wings, and on a Hybrid RANS/LES Approach". 1 st AFOSR International Conference on DNS/LES (invited), Aug. 1997.

[4] Huang, P. G., Coleman, G. N., and Bradshaw, P. "Compressible Turbulent Channel Flow - a Close Look Using DNS Data". AIAA Paper 95-0584, Jan. 1995.

[5] Gatski, T. B. and Erlebacher, G. "Numerical Simulation of a Spatially Evolving Supersonic Turbulent Boundary Layer". NASA Technical Report TM2002-211934, 2002.

[6] Spalding, D. B. "Mixing and Chemical Reaction in Confined Turbulent Flames". In Thirteenth Symposium (International) on Combustion, pages 649657, 1971.

[7] Magnussen, B. F. and Hjertager, B. H. "On Mathematical Modeling of Turbulent Combustion with Special Emphasis on Soot Formation and Combustion". In Sixteenth Symposium (International) on Combustion, pages 719-729, 1976.

[8] Kollman, W. "The PDF Approach to Turbulent Flow". Theoretical Computational Fluid Dynamics, Vol. 1:249-285, 1990.

[9] Pope, S. B. "PDF Methods for Turbulent Reactive Flows". Progress in Energy Combustion Science, Vol. 11:119-192, 1985.

[10] Pope, S. B. "A Monte Carlo Method for the PDF Equations of Turbulent Reactive Flow". Combustion Science and Technology, Vol. 25:159-174, 1980.

[11] Baldwin, B. S. and Lomax, H. "Thin Layer Approximation and Algebraic Model for Separated Turbulent Flows". AIAA Paper 78-0257, Jan. 1978.

[12] Jones, W. P. and Launder, B. E. "The Prediction of Laminarization with a Two Equation Model of Turbulence". International Journal of Heat and Mass Transfer, Vol. 15:301-314, 1972.

[13] Wilcox, D. C. Turbulence Modeling for CFD. DCW Industries, Inc., 2nd edition, 1998.

[14] Menter, F. R. "Zonal Two Equation $k$ - $\omega$ Models for Aerodynamic Flows". AIAA Paper 93-2906, July 1993.

[15] Robinson, D. F. and Hassan, H. A. "A TwoEquation Turbulence Closure Model for Wall Bounded and Free Shear Flows". AIAA Paper 962057, June 1996. 
[16] Durbin, P. A. "Near-Wall Turbulence Closure Modeling Without Damping Functions". Theoretical and Computational Fluid Dynamics, Vol. 3(1):1$13,1991$.

[17] Baldwin, B. S. and Barth, T. J. "A One-Equation Turbulence Transport Model for High Reynolds Number Wall-Bounded Flows". AIAA Paper 910610, Jan. 1991.

[18] Spalart, P. R. and Allmaras, S. R. "A One-Equation Turbulence Model for Aerodynamic Flows". AIAA Paper 92-0439, Jan. 1992.

[19] Launder, B. E., Reece, G. J., and Rodi, W. "Progress in the Development of a Reynolds-Stress Turbulence Closure". Journal of Fluid Mechanics, Vol. 68:537-566, 1975.

[20] Speziale, C. G., Sarkar, S., and Gatski, T. B. "Modelling the Pressure-Strain Correlation of Turbulence: An Invariant Dynamical Systems Approach". Journal of Fluid Mechanics, Vol. 227:245-272, 1991.

[21] Craft, T. J. and Launder, B. E. "A Reynolds Stress Closure Designed for Complex Geometries". International Journal of Heat and Fluid Flow, Vol. 17:245-254, 1996.

[22] Mellor, G. L. and Herring, H. J. "A Survey of the Mean Turbulent Field Closure Models". AIAA Journal, Vol. 11:590-599, 1973.

[23] Daley, B. J. and Harlow, F. H. "Transport Equations of Turbulence". Physics of Fluids, Vol. 13:2634 $2649,1970$.

[24] Hanjalic, K. and Launder, B. E. "A Reynolds Stress Model of Turbulence and its Application to Thin Shear Flows". Journal of Fluid Mechanics, Vol. 52:609-638, 1972.

[25] Rodi, W. "A New Algebraic Relation for Calculating the Reynolds Stresses". ZAMM, Vol. 56:219 $221,1976$.

[26] Pope, S. B. "A More General Effective Viscosity Hypothesis". Journal of Fluid Mechanics, Vol. 72:331-340, 1975.

[27] Taulbee, D. B. "An Improved Algebraic Reynolds Stress Model and Corresponding Nonlinear Stress Model". Physics of Fluids, Vol. 4:2555-2561, 1992.

[28] Gatski, T. B. and Speziale, C. G. "On Explicit Algebraic Stress Models for Complex Turbulent Flows". Journal of Fluid Mechanics, Vol. 254:59-78, 1993.
[29] Davis, D. O. and Gessner, F. B. "Further Experiments on Supersonic Turbulent Flow Development in a Square Duct". AlAA Journal, Vol. 27(8):10231030, Aug. 1989.

[30] Rumsey, C. L. and Gatski, T. B. "Recent Turbulence Model Advances Applied to Multielement Airfoil Computations". AIAA Paper 2000-4323, Aug. 2000.

[31] Speziale, C. G. "On Nonlinear $k-\ell$ and $k-\varepsilon$ Models of Turbulence". Journal of Fluid Mechanics, Vol. 178:459-475, 1987.

[32] Shih, T.-H., Zhu, J., and Lumley, J. L. "A New Reynolds Stress Algebraic Equation Model”. Computational Methods in Applied Mechanics and Engineering, Vol. 125:287-302, 1995.

[33] Craft, T. J., Launder, B. E., and Suga, K. "Development and Application of a Cubic Eddy-Viscosity Model of Turbulence". International Journal of Heat and Fluid Flow, Vol. 17:108-115, 1996.

[34] Gatski, T. B. "Turbulence Modeling for Aeronautical Flows". VKI Lecture Series, von Karman Institute for Fluid Dynamics, Nov. 2003.

[35] Brown, G. L. and Roshko, A. "On Density Effects and Large Structure in Turbulent Mixing Layers". Journal of Fluid Mechanics, Vol. 64:775-816, 1974.

[36] Battaglia, F. and Givi, P. "Direct Lagrangian Simulations of a Mixing Layer by the Transport-Element Method". Journal of Non-Equilibrium Thermodynamics, Vol. 18:173-194, 1993.

[37] Ballal, D. R., Chen, T. H., and Yaney, P. P. "An Experimental Investigation of Turbulent Mixing Using Rotational Raman Spectroscopy”. AIAA Paper 85$1105,1985$.

[38] Calhoon, W. H., Arunajatesan, S., and Dash, S. M. "Heat Release and Compressibility Effects on Planar Shear Layer Development". AIAA Paper 20031273, Jan. 2003.

[39] Schetz, J. A. Boundary Layer Analysis. Prentice Hall, 1993.

[40] Reynolds, A. J. "The Prediction of Turbulent Prandtl and Schmidt Numbers". International Journal of Heat and Mass Transfer, Vol. 18:1055-1069, 1975.

[41] Chidambaram, N., Dash, S. M., and Kenzakowski, D. C. "Scalar Variance Transport in the Turbulence Modeling of Propulsive Jets". AIAA Paper 99-0235, Jan. 1999. 
[42] Abe, K., Kondoh, T., and Nagano, Y. "A New Turbulence Model for Predicting Fluid Flow and Heat Transfer in Separating and Reattaching Flows - II. Thermal Field Calculations". International Journal of Heat and Mass Transfer, Vol. 38(8):1467-1481, 1995.

[43] Riggins, D. W., Mao, M., Bittner, R. D., McClinton, C. R., and Rogers, R. C. "Numerical Modeling of Normal Fuel Injection : Effect of Turbulent Schmidt Number". NASP Report, March 1989. Ref. WBS 2.4.09.

[44] Guo, Y., He, G., and Hsu, A. T. "The Development of a Variable Schmidt Number Model for Jetin-Crossflows Using Genetic Algorithms". AIAA Paper 99-0671, Jan. 1999.

[45] Sturgess, G. J. and McManus, K. R. "Calculations of Turbulent Mass Transport in a Bluff-Body Diffusion-Flame Combustor". AIAA Paper 840372, Jan. 1984.

[46] Baurle, R. A., Alexopoulos, G. A., and Hassan, H. A. "Analysis of Supersonic Combustors with Swept Ramp Injectors". Journal of Propulsion and Power, Vol. 3:327-328, March-April 1997.

[47] Baurle, R. A. and Eklund, D. R. "Analysis of DualMode Hydrocarbon Scramjet Operation at Mach 4-6.5". Journal of Propulsion and Power, Vol. 18:990-1002, Sept.-Oct. 2002.

[48] Eklund, D. R., Baurle, R. A., and Gruber, M. R. "Computational Study of a Supersonic Combustor Fueled by an Aerodynamic Ramp Injector". AIAA Paper 2001-0379, Jan. 2001.

[49] Moss, J. B. "Simultaneous Measurements of Concentration and Velocity in an Open Premixed Turbulent Flame". Combustion Science and Technology, Vol. 22:115-129, 1980.

[50] Libby, P. A. and Bray, K. N. C. "Counter Gradient Diffusion in Premixed Turbulent Flames". AIAA Paper 80-0013, Jan. 1980.

[51] Nishiki, S., Hasegawa, T. and Himeno, R. "Transport Properties in Fully Developed Turbulent Premixed Flames". High Performance Computing in RIKEN 2000 Review No. 40, Oct. 2000.

[52] Adumitroaie, V. "Quasi-Explicit Algebraic Turbulence Closures for Compressible Reacting Flows". $\mathrm{PhD}$ thesis, State University of New York at Buffalo, June 1997.
[53] Bilger, R. W. "Turbulent Flows with Nonpremixed Reactants". In P. A. Libby and F. A. Williams, editors, Turbulent Reacting Flow, pages 65-113. Springer-Verlag, 1980.

[54] Gaffney, R. L., White, J. A., Girimaji, S. S., and Drummond, J. P. "Modeling Turbulent/Chemistry Interactions Using Assumed PDF Methods". AIAA Paper 92-3638, July 1992.

[55] Gaffney, R. L., White, J. A., Girimaji, S. S., and Drummond, J. P. "Modeling Temperature and Species Fluctuations in Turbulent, Reacting Flow". Computing Systems in Engineering, Vol. 5(2):117133, 1994.

[56] Baurle, R. A. and Hassan, H. A. "Modeling of Turbulent Supersonic $\mathrm{H}_{2}$-Air Combustion With A Multivariate $\beta$ PDF”. AIAA Paper 93-2198, June 1993.

[57] Baurle, R. A., Hsu, A. T., and Hassan, H. A. "Comparison of Assumed and Evolution PDF's in Supersonic Turbulent Combustion Calculations". AIAA Paper 94-3180, June 1994.

[58] Baurle, R. A., Hsu, A. T., and Hassan, H. A. "Assumed and Evolution PDF's in Supersonic Turbulent Combustion Calculations". Journal of Propulsion and Power, Vol. 11:1132-1138, Nov. 1995.

[59] Möbus, H., Gerlinger, P., and Brüggemann, D. "Comparison of Eulerian and Lagrangian Monte Carlo PDF Methods for Turbulent Diffusion Flames". AIAA Paper 2000-0188, Jan. 2000.

[60] Gerlinger, P. "Investigation of an Assumed PDF Approach for Finite-Rate Chemistry". AIAA Paper 2002-0166, Jan. 2002.

[61] Baurle, R. A. and Girimaji, S. S. "An Assumed PDF Turbulence-Chemistry Closure with Temperature-Composition Correlations". Combustion and Flame, Vol. 134:131-148, July 2003.

[62] Cheng, T. S., Wehrmeyer, J. A., Pitz, R. W., Jarrett, O. Jr., and Northam, G. B. "Finite-Rate Chemistry Effects in a Mach 2 Reacting Flow". AIAA Paper 91-2320, June 1991.

[63] Girimaji, S. S. "A Simple Recipe for Modeling Reaction-Rates in Flows with Turbulent Combustion”. AIAA Paper 91-1792, June 1991. 


\section{FIGURES}

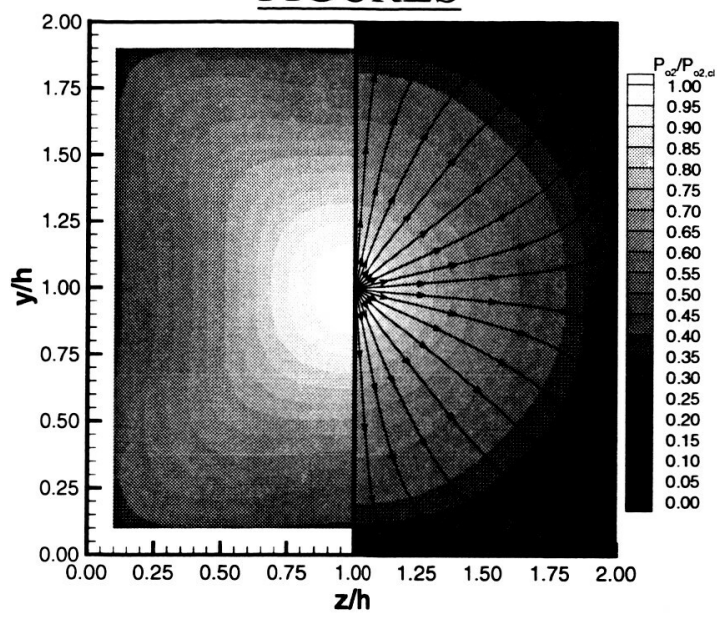

Figure 1: Measured (left) and computed ( $k$ - $\omega$ with Boussinesq) pitot pressure profiles at $\mathrm{x} / \mathrm{h}=40$

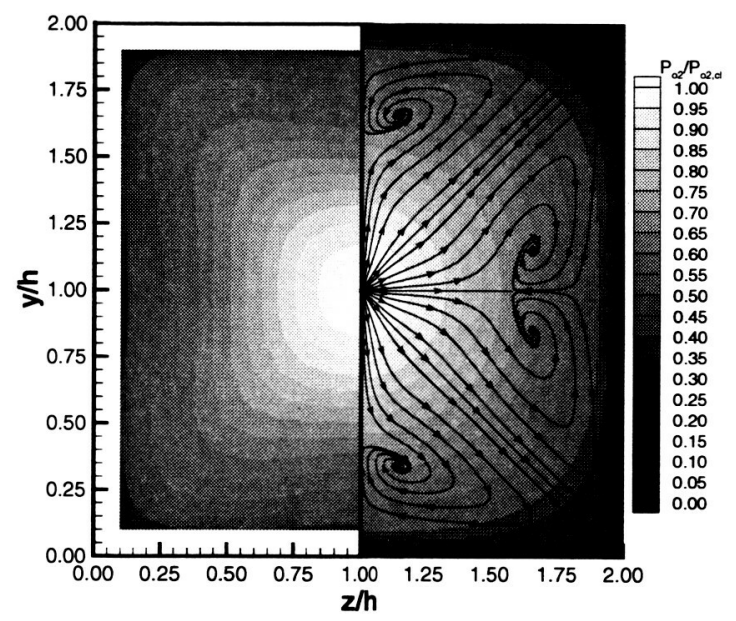

Figure 2: Measured (left) and computed (explicit algebraic Reynolds stress) pitot pressure profiles at $\mathrm{x} / \mathrm{h}=40$

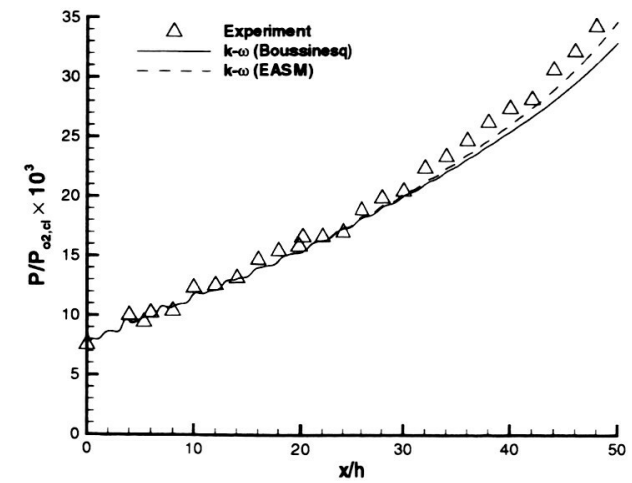

Figure 3: Measured and computed wall pressure distributions along the center of the duct 


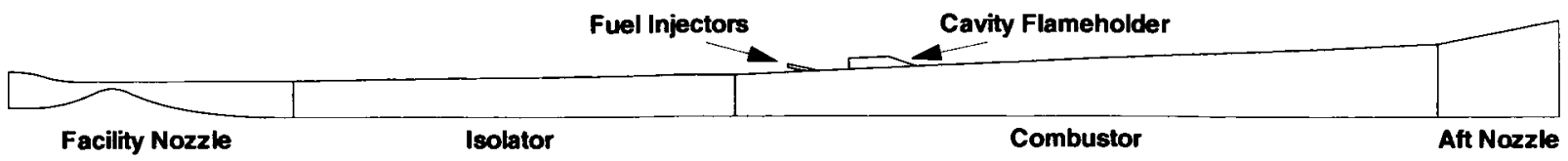

Figure 4: Schematic of facility flowpath
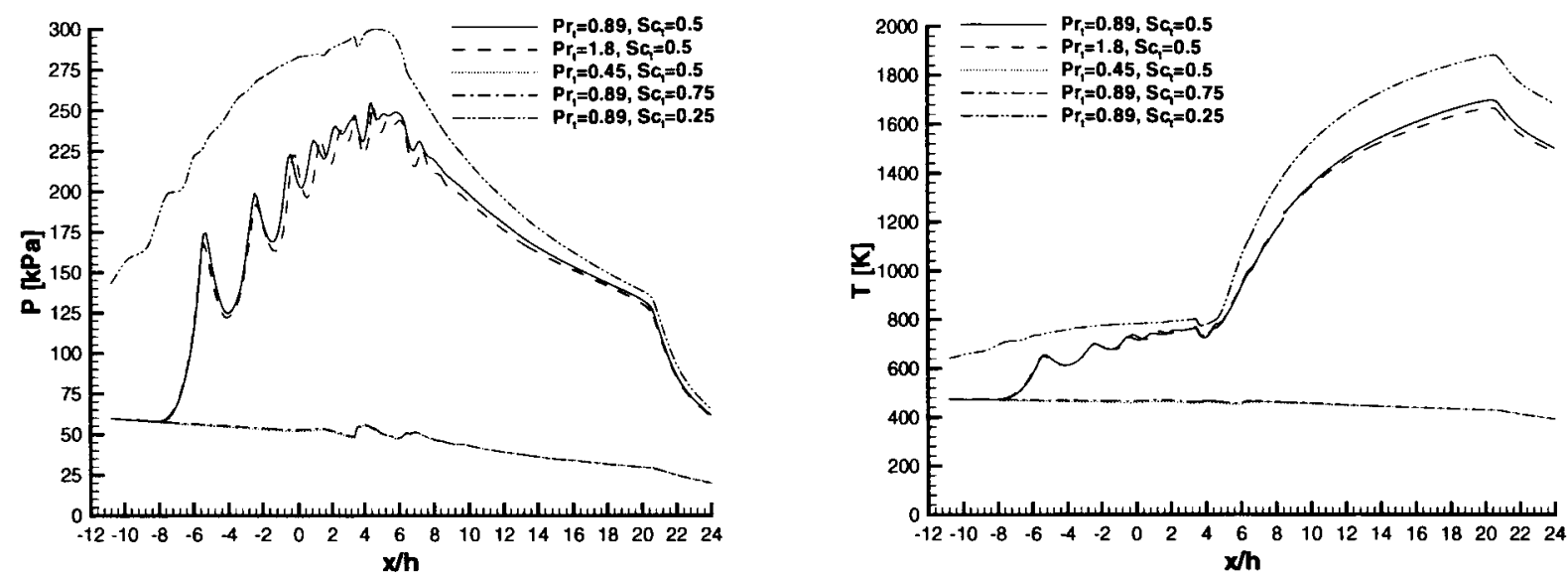

Figure 5: Mass-flux weighted pressure and temperature at the Mach 4.0 flight condition
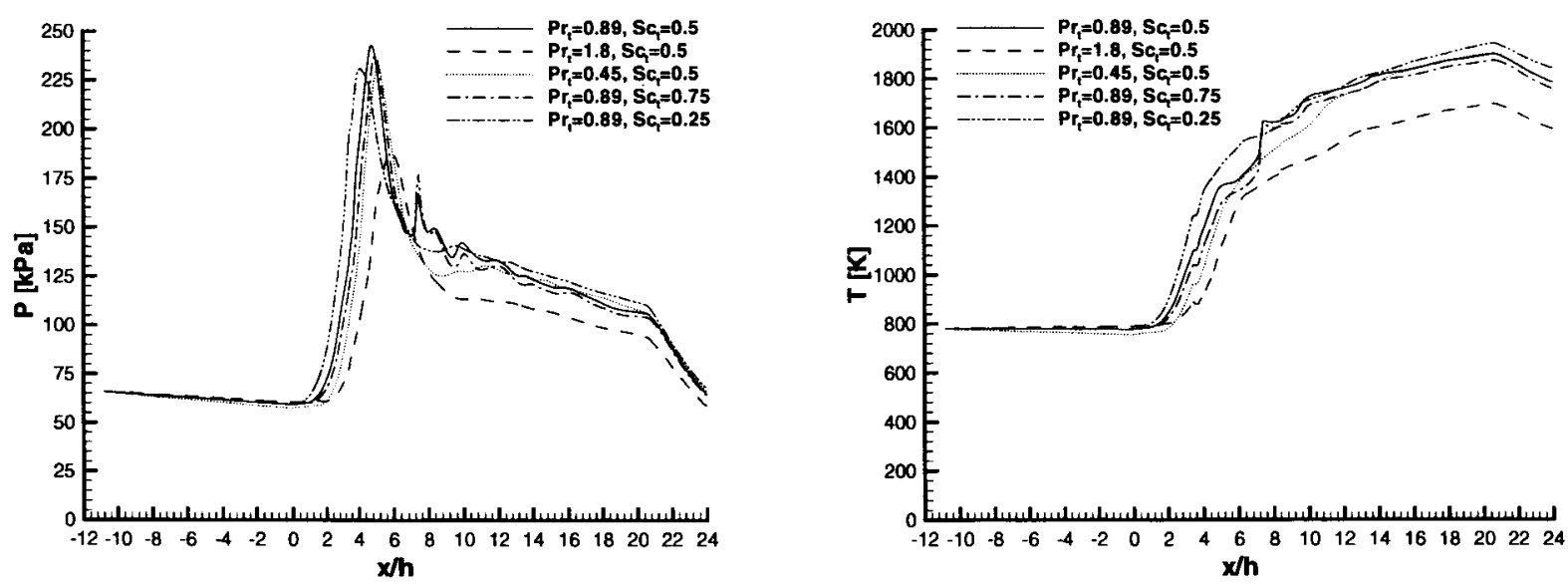

Figure 6: Mass-flux weighted pressure and temperature at the Mach 6.5 flight condition 

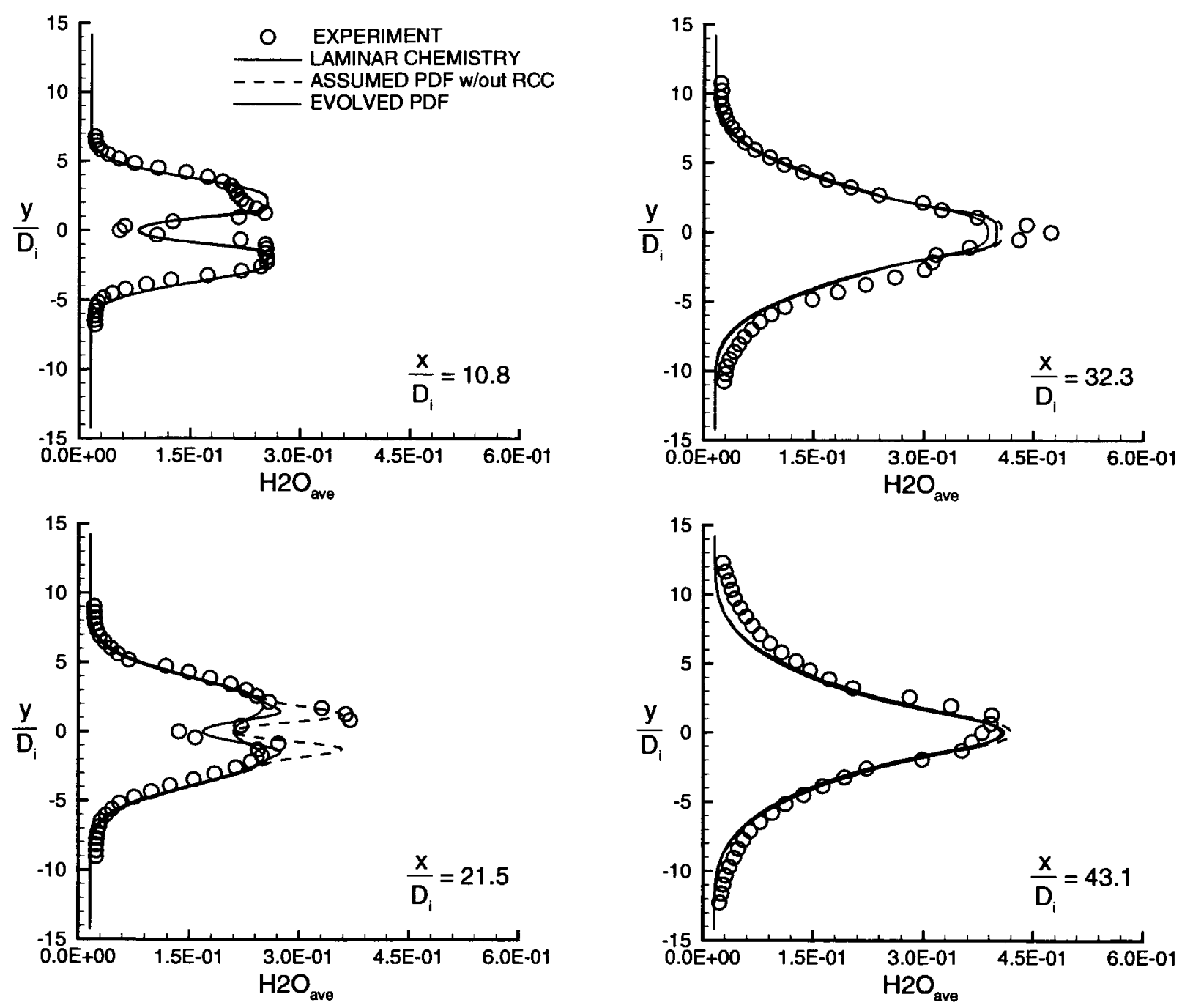

Figure 7: Mean $\mathrm{H}_{2} \mathrm{O}$ mole fraction comparisons with measurements (Cheng et al.) obtained from laminar chemistry, assumed PDF, and evolved PDF models 